\title{
ENTEROLOBIUM GLAZIOVII (BENTH.) MESQUITA, COMB. NOV. ET "STATUS NOVUM" PARA AS REGIÕES SUDESTE E NORDESTE DO BRASIL ${ }^{1}$
}

\author{
Antônio de L. Mesquita ${ }^{2}$
}

Recebido em 30-01-92. Aceito em 22-08-93.

\begin{abstract}
RESUMO - (Enterolobium glaziovii [Benth.] Mesquita, comb. nov. et "status novum" para as regiões Sudeste e Nordeste do Brasil). Eleva-se Enterolobium schomburgkii var. glaziovii Benth. a categoria de espécie, estabelecendo Enterolobium glaziovii (Benth.) Mesquita, "status novum" para as regiões Sudeste e Nordeste do Brasil. Tal resultado está baseado em estudo de coleções herborizadas, em observações de campo, em estudo comparativo de frutos, sementes embriões e na análise de padrões de distribuição geográfica. Apresenta-se uma descrição completa da espécie e tabela comparativa dos caracteres diferenciais entre esta e a espécie próxima $E$. schomburgkii (Benth.) Benth.
\end{abstract}

Palavras-chave: Enterolobium glaziovii, estudo morfológico, distribuição geográfica, Brasil.

ABSTRACT - (Enterolobium glaziovii [Benth.] Mesquita, "comb. nov. et status novum" to Southeastern and Northeastern). The author establishes Enterolobium glaziovii (Benth.) Mesquita, "status novum" to Southeastern and Northeastern of Brazil, based on herbarium collection, fieldwork, as well as in a morphological study of fruits, seeds and patterns of geographical distribution. A complete description of the species and a table of differential characters with the related species E. schomburgkii are presented.

Key-words: Enterolobium glaziovii, morphological study, geographical distribution, Brazil.

\section{Introdução}

Como parte da revisão taxonômica do gênero Enterolobium Mart. (Leguminosae Mimosoideae) para a região Neotropical, foi registrado um "status novum" para os estados do Rio de Janeiro, Bahia, Espírito Santo e Minas Gerais.

1 - Trabalho apresentado no XLI Congresso Nacional de Botânica, Fortaleza-Ceará. 1990.

2 - Instituto de Tecnologia da Amazônia-UTAM-CEPEF. Dept ${ }^{\circ}$ de Manejo Florestal Av. Darcy Vargas, 1200. Manaus-AM. 69050. Bolsista da CAPES. 
Bentham (1876) descreveu pela primeira vez este táxon, como E. schomburgkii var. glaziovii, coletado por Glaziou, no Rio de Janeiro.

Glaziou (1906), em seu trabalho sobre as plantas coletadas no Brasil central, registra a ocorrência desta variedade para o Rio de Janeiro, e cita em sua lista de material coletado, os exemplares 1148, 626, 1494 e 11935.

Barroso (1965), em seu estudo sobre as leguminosas da Guanabara, registra a ocorrência desta espécie para as Matas da Tijuca e Campo Grande.

Lewis (1987) cita a ocorrência desta espécie para a flora da Bahia, como $E$. schomburgkii.

Bentham (1876) ao propor E. schomburgkii var. glaziovii, ressalta o fato de não ter analisado suas flores, assim como coloca em dúvida a validade infraespecífica do táxon, 0 que nos levou a um estudo taxonômico e nomenclatural, fornecendo-nos os elementos necessários para o reconhecimento deste novo táxon.

\section{Material e métodos}

O material básico para esta pesquisa foi obtido através de uma vasta coleção de exsicatas, provenientes dos herbários BHMG, BM, C, GH, HITAM, IPA, LE, K, MG, $\mathrm{NY}, \mathrm{P}, \mathrm{R}, \mathrm{RB}$ e $\mathrm{S}$, incluindo os isolectótipos, e também observações de campo, juntamente com o estudo comparativo da morfologia do fruto, semente e embrião, e do padrão de distribuição geográfica.

\section{Resultados e discussão}

Enterolobium glaziovii (Benth.) Mesquita, comb. nov. et stat. nov.

Tipo. Brasil: Habitat prope Rio de Janeiro, Glaziou 1148, folia com fructu único, lectótipo, P! (aqui designado); isolectótipos, BM!, BR!, C!; foto do isolectótipo, NY!.

Figura 1: A-F

Basiônimo: Enterolobium schomburgkii var. glaziovii Benth., in Martius, Fl. Bras. 15(2): 457. 1876.

Sinônimo: Enterolobium lutescens Martius, in Corrêa, Dic. Pl. Úteis Exót. Cult. Brasil 3:32. 1926, nomen nudum.

Árvores de 3-30 m de altura, $18 \mathrm{~cm}$ de diâmetro, copa densa, córtex acinzentado. Folhas com ca. de $34 \mathrm{~cm}$ de comprimento, com 20-30 pares de pinas; ráque ferrugíneovilosa, face superior em forma triangular, lustrosa, face inferior canaliculada; pecíolo com 2-3 cm de comprimento, com glândula elíptico-transversa na base; foliólulos linearfalcados, lanceolados, assimétricos, 40-80 pares por pinas, o terminal com ca. de $3 \mathrm{~mm}$ de comprimento, $2 \mathrm{~mm}$ de largura, o mediano com ca. de $4 \mathrm{~mm}$ de comprimento, $2 \mathrm{~mm}$ de largura, margem inteira, densamente pilosa, ápice agudo, base oblíquo-irregular, nervação inconspícua, nervura central atingindo as margens. Glomérulos fasciculares, axilares, homomórficos, dispostos em inflorescências longamente pedunculadas com ca. de 40 $\mathrm{mm}$ de comprimento, densos (35-70 flores), pedicelo com 1-2 mm de comprimento; 
flores ferrugíneo-vilosas; cálice tubuloso, ferrugíneo-viloso, com 2-4 mm de comprimento, 5-laciniado; lacínias com $0,5 \mathrm{~mm}$ de comprimento; corola ferrugíneo-vilosa, infundibuliforme, com ca. de $5 \mathrm{~mm}$ de comprimento, 5-laciniada, lacínias com ca. de 1 $\mathrm{mm}$ de comprimento; androceu, com ca. de $14 \mathrm{~mm}$ de comprimento, tubo estaminal exserto; estames numerosos; gineceu de ovário alvo, viloso com ca. de $1 \mathrm{~mm}$ de comprimento; estilete com ca. de $12 \mathrm{~mm}$ de comprimento; óvulos ca. de 23-29. Fruto lenhoso, contorcido, com 8-10 de comprimento, $4-5 \mathrm{~cm}$ de largura, com a presença de 23 sob o pedúnculo, margem sinuosa, base auricular, obliquo-reentrante, replum espesso, epicarpo lenhoso, negro, superficie lustrosa, ornamentação rugosa, textura glabra, região seminífera externamente indistinta, região mediana ligeiramente depressa; mesocarpo creme, fibroso; endocarpo indiferenciado; pedúnculos com ca. de $4 \mathrm{~cm}$ de comprimento; semente oval, com ca. de $0,1 \mathrm{~cm}$ de comprimento, $0,4 \mathrm{~cm}$ de largura, amarelo-enxofre, testa fina, monocromada, pleurograma diferenciado, com coloração clara, lanceolado; aréola com linha fratural presente; embrião com cotilédones ovais, com ca. de $1 \mathrm{~cm}$ de comprimento; eixo embrionário deflexo; eixo hipocótilo-radícula exposto, com ca. de 4 mm de comprimento; plúmula bem desenvolvida, diferenciada em pinas.

Distribuição: E. glaziovii apresenta distribuição restrita, predominantemente às regiões Nordeste (Bahia) e Sudeste do Brasil, nos estados do Rio de Janeiro, Minas Gerais e Espírito Santo (Figura 2). No Rio de Janeiro, encontramos esta espécie na Floresta da Tijuca, Alto da Boa Vista, Horto Florestal, próximo à estrada D. Castorinha, que dá acesso à Vista Chinesa. No Espírito Santo, sua ocorrência foi registrada para a mata semidecidual dos municípios de Linhares, Reserva florestal da CVRD e Santa Teresa. Para o estado de Minas Gerais, verificou-se sua ocorrência no município de Tombos. Sua ocorrência também está assinalada, através de uma única coleta, para 0 Nordeste do Brasil, estado da Bahia, localidade Fazenda das Pedras, coletada por M. Filho \& Rizzini, depositada nos herbários RB e IPA. Barroso (1965), em seu estudo sobre as leguminosas da Guanabara, registra a ocorrência desta espécie para as matas da Tijuca e Campo Grande. Lewis (1987), cita a ocorrência desta espécie para a flora da Bahia, como E. schomburgkii.

Taxonomia e Nomenclatura: A espécie E. glaziovii caracteriza-se por apresentar o fruto lenhoso, com epicarpo rugoso, sementes ovais, com apenas $1 \mathrm{~cm}$ de comprimento, 0 que é suficiente para distinguí-la de todas as demais espécies do gênero. Além disto, $E$. glaziovii pode ainda ser reconhecida por apresentar ramos e flores totalmente cobertos por indumento ferrugíneo-viloso.

Enterolobium glaziovii apresenta estreita afinidade com E. schomburgkii, principalmente com relação ao número, tamanho e forma dos foliólulos. Porém, as diferenças entre elas consistem em vários caracteres, a nível morfológico, fitogeográfico e habitat, conforme pode ser observado na Tabela 1.

Bentham (1876) descreveu este táxon como E. schomburgkii var. glaziovii, baseando-se nos seguintes síntipos: Rio de Janeiro: Glaziou 626 (folha estéril) e Glaziou 1148 (folha com único fruto). Este mesmo autor, ao descrevê-lo, ressalta o fato de não ter visto as suas flores, como também coloca em dúvida a posição infraespecífica do táxon. 
Côrrea (1926) registrou um fato muito significativo sobre $E$. schomburgkii var. glaziovii. Segundo 0 autor, botânicos antigos como Freire Alemão e outros, referem 0 táxon validamente publicado por Bentham (1876), como uma nova espécie, E. lutescens Martius. Na verdade, esta espécie não foi validamente publicada e, portanto, se constitui em um nome nudum e é um sinônimo de $E$. glaziovii.

O epíteto desta espécie homenageia o botânico francês Auguste François Marie Glaziou.

Tipificação: Bentham (1876), ao descrever o táxon, não mencionou o herbário em que estariam depositados os sintipos das coleções: Glaziou 626 e 1148. Desta forma, na escolha dos lectótipos, deveria ser dada preferência ao herbário de Kew (K), no qual trabalhou Bentham. Entretanto, consultamos os botânicos Charles Stirton e Gwilym Lewis, do herbário de Kew, os quais nos informaram não terem sido encontradas as coleções de Glaziou no citado herbário. Como Glaziou (1906) citou, porém, em sua lista de plantas coletadas no Brasil central, os exemplares 626 e 1148, para as coleções da variedade $E$. schomburgkii var. glaziovii, fizemos consulta ao herbário de Paris, onde se encontra 0 material coletado por Glaziou, e o resultado foi positivo. Assim, elegemos como lectótipo o exemplar 1148, e como paralectótipo o exemplar 626 de Glaziou, depositados no herbário de Paris. Devemos ressaltar o fato que a análise do lectótipo (herbário P) e dos isolectótipos (herbários BM, BR e C), juntamente com observações de campo, aliadas ao estudo da morfologia da flor e do fruto, forneceram-nos elementos necessários para 0 reconhecimento deste táxon.

Geograficamente esta espécie apresenta distribuição alopátrica com E. schomburgkii e simpátrica com E. monjollo (Vell.) Mart.

Material examinado: Brasil. Bahia: Fazenda das Pedras, dez. 1956 (fr), M. Filho \& Rizzini s/n (RB, IPA). Espírito Santo: Santa Teresa, nov. 1948 (fr), A. Ruschi s/n (RB); Linhares, Reserva Florestal da CVRD, 27 nov. 1978 (bot, fl), I. A. S. 033.78 (CVRD, MG, RB); ibid., 30 nov. 1972 (fl), J. Spada 107 (CVRD, RB). Minas Gerais: Tombos (fazenda da cachoeira), jul. 1935 (fr), Barreto 1723 (BHMG, IPA, RB). Rio de Janeiro: (est), A. Glaziou 626 (isolectótipos, BR!, RB!, C!); estrada que dá acesso à Vista Chinesa, 29 fev. 1986 (fl, fr), A. Mesquita, H. de Lima, J. Gomes, 908 (RB, HITAM 2610); ibid., 27 mar. 1985 (fr), J. Gomes 48 (RB); ibid, 20 nov. 1962 (fl), C. Angeli 340 (GUA, HRB); 1880 (bot, fl), A. Glaziou 11935 (LE); maio 1881 (fl), A. Glaziou 11931 (GH); (est), A. Glaziou 11938 (BM, RB); (est), A. Glaziou 1194 (BM, C); (est), A. Glaziou 1494 (BR, C); (fl,fr), A. Glaziou 1867 (BR); set. 1880 (fl), A. Glaziou 11937 (GH, NY); Corcovado, 1874 (fr), M. Clausen 2477 (S); (fl), F. Alemão (RB 5224); Botafogo, set. 1921 (fl), J. Kuhlman s/n (RB, S); Horto Florestal, out. 1926 (fl), J. Kuhlman \& A. Ducke s/n (RB, S); 1874 (fr), H. Mosên 2477 (S); Avelar, nov. 1925 (fr), G. Nunes 11 (R, RB 20175); 1931 (fr), G. Nunes s/n (R 24119); Matas da Tijuca, nov. 1926 (fl), Horto Florestal s/n (RB); Horto Florestal, próximo à estrada D. Castorinha, fev. 1930 (fl), P. Rosa \& C. Lage s/n (RB); Alto da Boa Vista, out. 1958 (fr), E. Pereira s/n (RB); Entre Mesa do Imperador e Alto da Boa Vista, fev. 1959 (fr), E. Pereira \& A. P. Duarte 4538 
(NY, RB); Campo Grande, Merdanha, sem coletor, s/d (fl), (R, RB 68580); Sem localidade exata, sem coletor, s/d (fr), (R 3550).

Habitat: E. glaziovii é nativa e restrita à Mata Atlântica, ocorrendo nas matas de encosta do Rio de Janeiro, mata de terra firme na Bahia e na floresta estacional remanescente da Mata Atlântica de Minas Gerais e Espírito Santo (Jesus, 1987).

Fenologia: Segundo Glaziou (1906), esta espécie floresce e frutifica nos meses de maio e novembro. Na estrada D. Castorinha, no Rio de Janeiro, coletamos E. glaziovii com flores e frutos em fevereiro, sendo que os frutos se encontravam em estádio jovem (Mesquita et al. 908). Entretanto, verificamos que esta espécie floresce e frutifica principalmente nos meses de junho a março.

Nomes Populares: Brasil. Rio de Janeiro: Orelha-de-negro, vinhático-cabeleira, angico-pedra, timbó, orelha-de-macaco, timboiba. Bahia: Tambor. Espírito Santo: Cobi. Segundo Glaziou (1906) esta espécie é também vulgarmente conhecida como Cabuvinhático.

Tabela 1 - Comparação dos caracteres diferenciais entre E. glaziovii e E. schomburgkii

\begin{tabular}{lll}
\hline \multicolumn{1}{c}{ Caracteres } & \multicolumn{1}{c}{ E. glaziovii } & E. schomburgkii \\
\hline Glomérulos & homomórficos & heteromórficos \\
Cálice/indumento & tubuloso/viloso & $\begin{array}{l}\text { campanulado (flor } \\
\text { central); infundibuliforme } \\
\text { (laterais/tomentoso }\end{array}$ \\
Epicarpo & rugoso & liso \\
Semente & oval & elíptica \\
& Restrita às regiões & $\begin{array}{l}\text { Ampla: América Central } \\
\text { e América do Sul (Brasil: } \\
\text { Amazônia, Maranhão e }\end{array}$ \\
Distribuição geográfica & $\begin{array}{l}\text { Sudeste e Nordeste } \\
\text { (Bahia) do Brasil }\end{array}$ & Piauí) \\
Habitat & Nativa da Mata Atlântica & Mata Amazônica
\end{tabular}




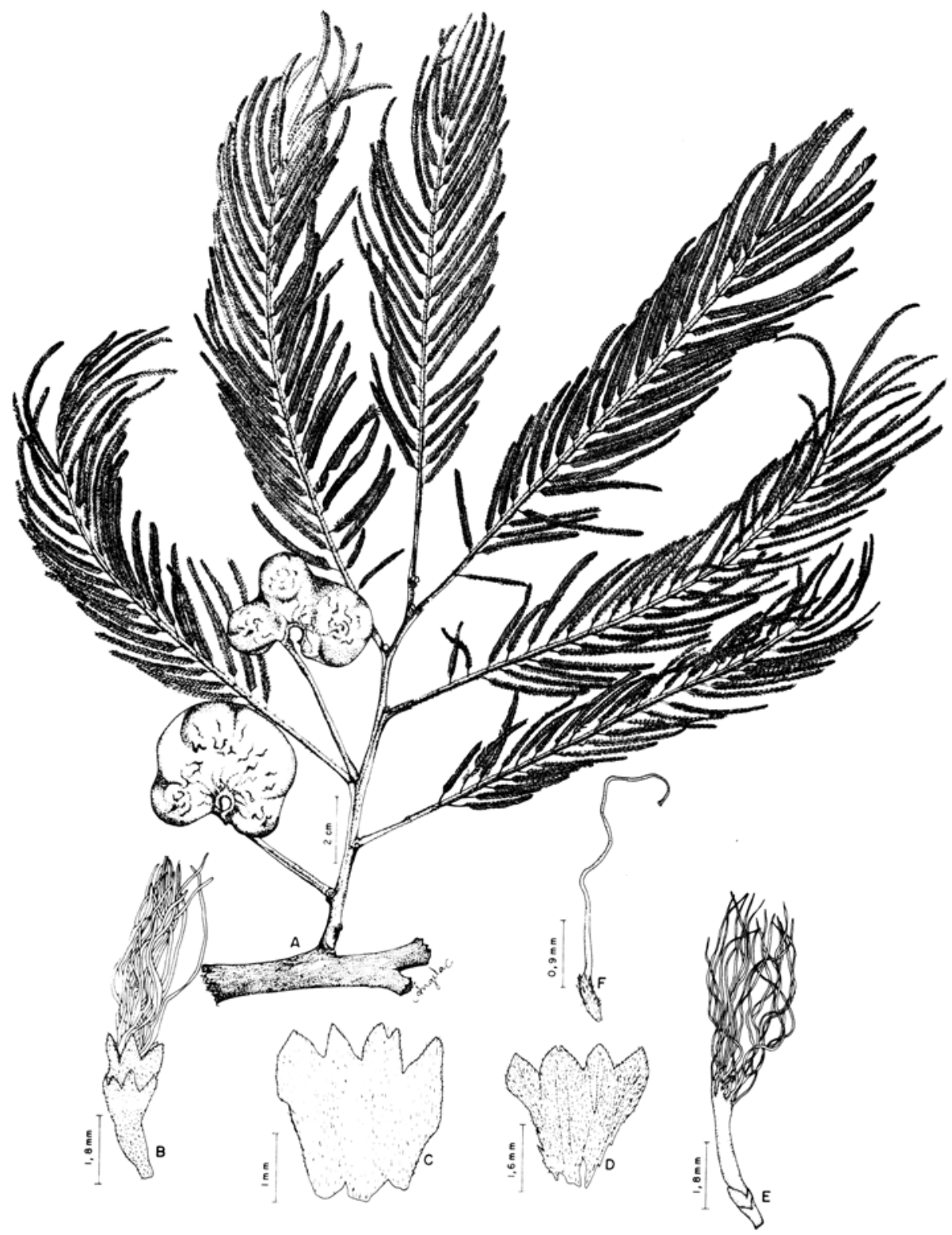

Figura 1 - E. glaziovii (Benth.) Mesquita: A. hábito e frutos; B. flor; C. corola aberta; D. cálice aberto; E. tubo estaminal; F. gineceu (A. L. Mesquita et al. 908) 


\section{Agradecimentos}

Ao Dr. Geraldo Mariz, à Dra. Graziela Maciel Barroso e ao Dr. Jorge Fontella pelas críticas e sugestões. À CAPES pela bolsa de estudo concedida na realização desta pesquisa.

\section{Referências Bibliográficas}

Barroso, G.M. 1965. Leguminosas da Guanabara. Arquivos do Jardim Botânico do Rio de Janeiro, Rio de Janeiro. 18: 109-182.

Bentham, G. 1876. Leguminosae-Mimosoideae. In C.F.P. Martius. Flora Brasiliensis. 15(2): 456-458.

Corrêa, M.P. 1926. Dicionário de plantas úteis do Brasil e das exóticas cultivadas. Rio de Janeiro. Ministério da Agricultura.

Glaziou, A.F.M. 1906. Planta brasiliae centralis a Glaziou lectae; liste des plantes du Brésil Central recueillies en 1861-1895. Mémoires Societté Botanique de França. 1(3): 1-661.

Jesus, R. M. de. 1987. Mata Atlântica de Linhares: aspectos florestais. In: Anais Seminário do Desenvolvimento Econômico e Impacto Ambiental e Áreas de Trópico Úmido Brasileiro, a experiência do CVRD. Rio de Janeiro. p. 36-71.

Lewis, G.P. 1987. Legumes of Bahia. Royal Botanic Gardens. Kew. 